\title{
Knowledge domain and emerging trends in chronic prostatitis/ chronic pelvic pain syndrome from 1970 to 2020: a scientometric analysis based on VOSviewer and CiteSpace
}

\author{
Sheng-Jing Liu ${ }^{1,2} \wedge$, Qing-He Gao ${ }^{1}$, Ying-Jun Deng ${ }^{1}$, Yin Zen ${ }^{3}$, Ming Zhao ${ }^{4}$, Jun Guo ${ }^{1}$ \\ ${ }^{1}$ Xiyuan Hospital of China Academy of Chinese Medical Sciences, Beijing, China; ${ }^{2}$ Graduate School of China Academy of Chinese Medical Sciences, \\ Beijing, China; ${ }^{3}$ Beijing Chinese Medicine Hospital Affiliated to Capital Medical University, Beijing, China; ${ }^{4}$ Beijing University of Chinese Medicine, \\ Beijing, China \\ Contributions: (I) Conception and design: SJ Liu; (II) Administrative support: J Guo; (III) Provision of study materials or patients: None; (IV) \\ Collection and assembly of data: YJ Deng, Y Zen, M Zhao; (V) Data analysis and interpretation: SJ Liu, QH Gao; (VI) Manuscript writing: All \\ authors; (VII) Final approval of manuscript: All authors. \\ Correspondence to: Jun Guo, MD. Xiyuan Hospital of China Academy of Chinese Medical Sciences, Beijing 100091, China. Email: guojun1126@126.com.
}

\begin{abstract}
Background: Chronic prostatitis/chronic pelvic pain syndrome (CP/CPPS) is a common urological disease, and research on CP/CPPS has increased over the past 50 years. However, few studies have statistically analyzed these publications. In this work, we conducted the knowledge domain and highlighted current research hotspots and emerging trends in CP/CPPS from 1970 to 2020 based on VOSviewer and CiteSpace.
\end{abstract}

Methods: Relevant original articles were obtained from the Web of Science (WoS) database between 1970 and 2020. VOSviewer and CiteSpace software were used to perform the analysis and visualization of scientific productivity and emerging trends.

Results: Our results show that the articles related to CP/CPPS have dramatically increased every year from 1 publication in 1970 to 111 publications in 2020. The USA dominated the field in all countries, and Queen's University (Canada) has more extensive cooperating relationships with other institutions. J. Curtis Nickel may have a significant influence on CP/CPPS research with more publications and cocitations. The fournal of Urology is the foremost productive journal and has the most citations of all the journals. A total of 11 major clusters were explored based on the reference cocitation analysis (RCA). Definition, incidence rate or clinical characteristics, etiology or pathogenesis, epidemiological studies (cross-sectional study and cohort study), clinical studies (inflammation, pain, lower urinary tract symptoms (LUTS), $\alpha$-blockers, antibiotic) and relationships with other diseases [benign prostatic hyperplasia (BPH), prostate cancer, sexual dysfunction] are the knowledge bases for CP/CPPS research. The treatment mode also changed gradually from antiinflammatory therapy to symptom improvement, and NIH-CPSI was taken as the evaluation criterion.

Conclusions: This scientometric study comprehensively reviewed publications related to CP/CPPS during the past 50 years using quantitative and qualitative methods, and the information provides some references for scholars to conduct further research on CP/CPPS.

Keywords: Chronic prostatitis/chronic pelvic pain syndrome (CP/CPPS); VOSviewer; CiteSpace; scientometric analysis

Submitted Oct 21, 2021. Accepted for publication Jan 04, 2022.

doi: 10.21037/apm-21-3068

View this article at: https://dx.doi.org/10.21037/apm-21-3068

$\wedge$ ORCID: 0000-0003-2828-0959. 


\section{Introduction}

Chronic prostatitis/chronic pelvic pain syndrome (CP/ CPPS), also known as chronic prostatitis (CP), nonbacterial prostatitis, or NIH Category III prostatitis, is a form of inflammation that can cause long-term pain or is extremely uncomfortable in the pelvic area or lower urinary tract symptoms (LUTS) (1). CP/CPPS is a prevalent condition in men worldwide, yet it is poorly understood $(2,3)$. CP/ CPPS is one of the most frustration diseases for doctors (4). A large amount of literature has been published for a better understanding of this complex disease. Literature combing is essential for a better overall understanding of the disease and provides a deeper perspective for practitioners (5).

Bibliometric analysis can quantify the impact of independent research results and the literature development of a research field or a specific disease and evaluate the tendencies of scientific research (6). Researchers can summarize the current situation and development trends of specific subjects and provide directions and ideas for future research by analyzing the characteristics of the literature, such as countries, institutions, journals, authors, keywords and references (6). Web of Science (WoS) is considered the optimal database for bibliometric analysis, and the current software for bibliometric analysis includes VOSviewer (7) (Leiden University, Leiden, The Netherlands) and CiteSpace (8) (Drexel University, Philadelphia, PA, USA). This study aims to use bibliometric methods to identify the contribution of countries, institutions and authors, milestone manuscripts, research themes, and emerging trends in the CP/CPPS research field.

\section{Methods}

\section{Data source and search strategy}

We searched WoS to collate CP/CPPS-related studies between 1970 and 2020. The database source was limited to Science Citation Index Expanded (SCIE) and publication types to "article", and the language of publications was limited to English. The main search terms were as described below: "chronic prostatitis/chronic pelvic pain syndrome", "chronic prostatitis", "chronic abacterial prostatitis", "chronic nonbacterial prostatitis", and "chronic prostatic inflammation". All eligible data from the WoS were downloaded for further analysis by scientometric tools.

\section{Statistical analysis}

Cleaning of the data was performed prior to analysis, and then we deleted meaningless keywords and words related to search terms and merged the same meaning keywords.

VOSviewer (1.6.16) was used to identify productive countries/regions, institutions, journals and authors, and the main cocited journals, authors and references and related visual networks were also constructed. CiteSpace (5.0. R2) was used to construct cluster analysis of high-frequency keywords based on the clustering function. We managed the data and analyzed the publication trends using Microsoft Office Excel 2019 (Microsoft Corporation, Redmond, WA, USA). The linear model $f(x)=a x+b$ was used to predict the number of studies in 2020. Variable $f(x)$ represents the number of studies, and $\mathrm{x}$ denotes the publication year. In the VOSviewer network maps, the size of the nodes reflects the number of studies or cooccurrence frequencies, the links between nodes represent the cooccurrence relationships, and the size of the links indicates the cooccurrence frequencies of two nodes. The impact factors (IFs) of the academic journals were collected from the 2020 Journal Citation Reports (JCR) (Clarivate Analytics, Philadelphia, PA, USA).

\section{Results}

\section{Annual growth trend of publications}

According to the WoS database, a total of 1,908 articles were published between 1970 and 2020, which were cited 31,723 times, and each article was cited 15.90 times on average. The model fitting curves of the growth trends of the accumulated number of publications are shown in Figure 1. The number of publications devoted to CP/CPPS has increased steadily over the last 50 years, and the linear fitting of publications shows a significant correlation. There was an evident increasing trend up to 2003, and nearly 100 new articles per year were published in the last 5 years.

\section{Contribution of countries and institutions}

A total of 1,908 publications on CP/CPPS were coauthored by 1,905 institutions from 71 countries/regions. The top 10 most productive countries and institutes regarding CP/CPPS are shown in Table 1. The USA was the foremost productive country, with 637 articles published and 24,305 citations. Of the top 10 active institutions, 6 were from the USA, indicating that broad studies have been conducted on CP/CPPS in the USA.

Countries $(34 / 71,47.9 \%)$ and institutions $(62 / 1,904$, 


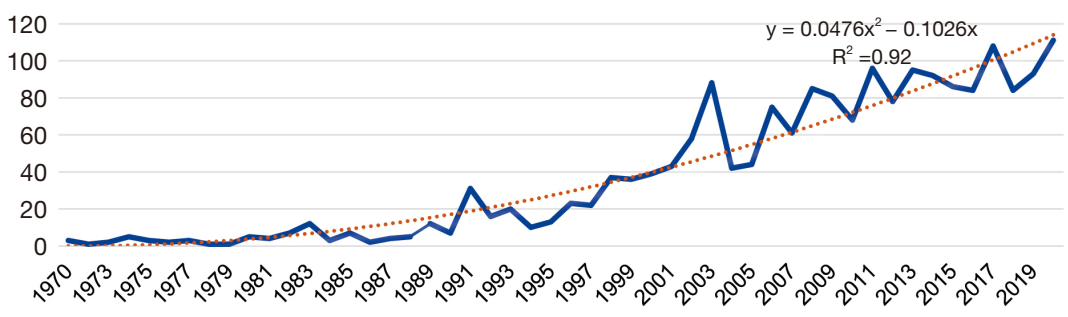

Figure 1 The output of articles and growth prediction of CP/CPPS research. The blue solid line represents the number of articles published between 1970 and 2020, and the yellow dotted line represents the linear fitting curve predicting the expected articles. CP/CPPS, chronic prostatitis/chronic pelvic pain syndrome.

Table 1 The top 10 countries and institutes contributed to publications about CP/CPPS

\begin{tabular}{|c|c|c|c|c|c|c|}
\hline Rank & Country/region & Documents & Citations & Institution/country & Documents & Citations \\
\hline 2 & China/Asia & 262 & 2,259 & Queens Univ/Canada & 93 & 5,498 \\
\hline 3 & Italy/Europe & 179 & 4,746 & Northwestern Univ/USA & 86 & 4,168 \\
\hline 4 & Germany/Europe & 128 & 3,734 & Univ Giessen/Germany & 51 & 1,631 \\
\hline 6 & South Korea/Asia & 87 & 1,120 & Univ Florence/Italy & 45 & 1,728 \\
\hline 7 & Turkey/Asia & 72 & 1,184 & Univ Penn/USA & 44 & 3,009 \\
\hline 8 & Japan/Asia & 68 & 875 & Anhui Med Univ/China & 33 & 359 \\
\hline 9 & England/Europe & 51 & 2,007 & Univ Michigan/USA & 30 & 1,177 \\
\hline
\end{tabular}

CP/CPPS, chronic prostatitis/chronic pelvic pain syndrome.

$3.3 \%$ ) with publication numbers greater than or equal to 10 $(\mathrm{T} \geq 10)$ were used to construct the co-occurrence network map, as shown in Figure $2 A, 2 B$. The figures show that the USA, Canada, Italy and China have larger sizes of nodes and thicker lines, indicating that they have more publications and more collaborations. In the institutions, University of Washington (USA) published the largest number of articles; however, Queen's University (Canada) had more extensive cooperating relationships with other institutions. According to the principle that the different colors represent different times, China is currently being produced considerable research in CP/CPPS.

\section{Authors' and journals' cooperation network}

A total of 1,908 articles were published by 7,538 authors in a collaborative effort. The top 10 most productive authors and their citations about CP/CPPS are shown in
Table 2. The authors $(42 / 7,540,0.6 \%)$ with a publication number greater than or equal to $10(\mathrm{~T} \geq 10)$ were used to construct the co-occurrence network map, as shown in Figure 2C. J. Curtis Nickel was the foremost productive author and had the most author collaborations, with 96 articles published and 5,650 citations.

Of 1,908 articles were published by 476 journals. The top 10 most productive journals and their citations about CP/CPPS are shown in Table 3. Journals (32/476, 6.7\%) with a publication number greater than or equal to 10 $(\mathrm{T} \geq 10)$ were used to construct the co-occurrence network map, as shown in Figure 2D. The fournal of Urology $\left(\mathrm{IF}^{2020}\right.$ $=7.450, \mathrm{Q} 1)$ is the foremost productive journal and had the most citations of all the journals. And the European Urology $\left(\mathrm{IF}^{2020}=20.097, \mathrm{Q} 1\right)$ is the journal with the highest IF on CP/CPPS. We further analyzed the keywords of articles published by the significant journals fournal of Urology and European Urology, as shown in the Figure 2E,2F. 
A

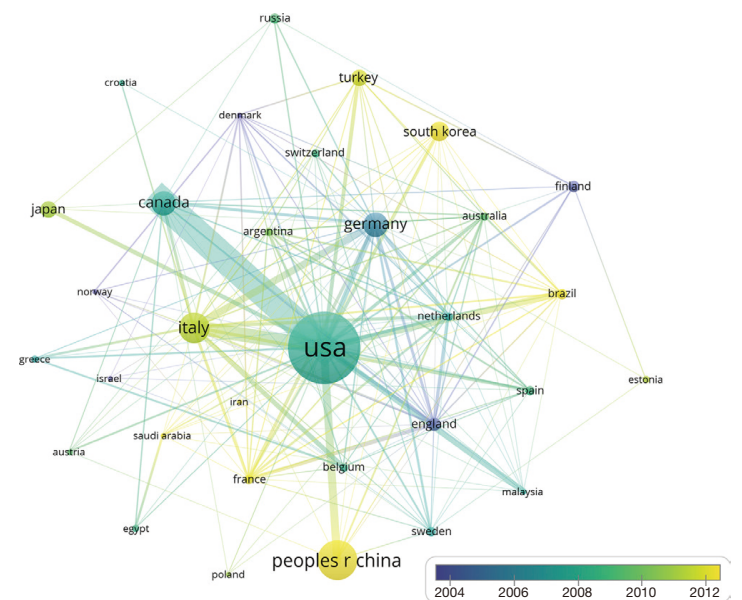

C

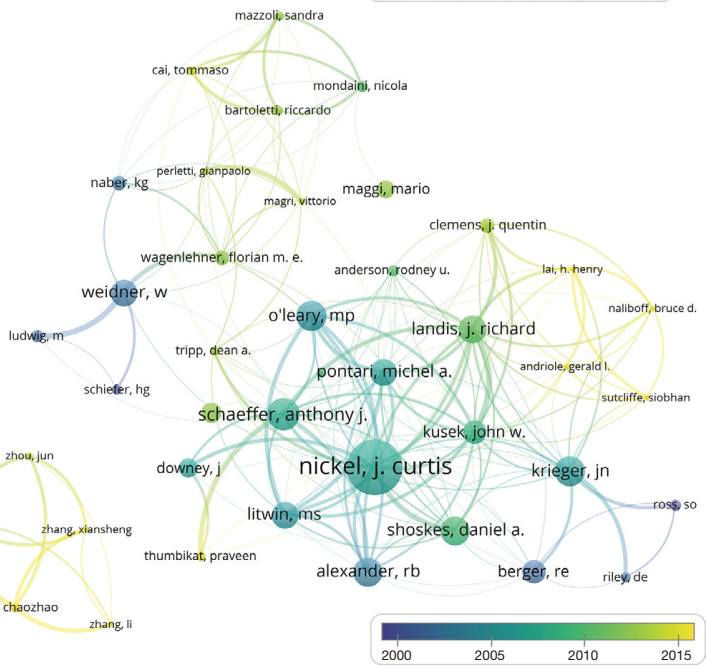

E

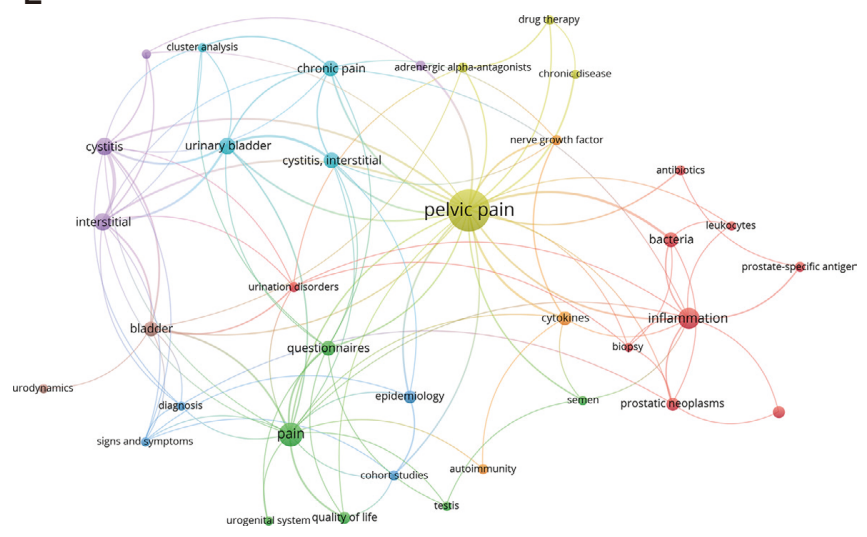

B
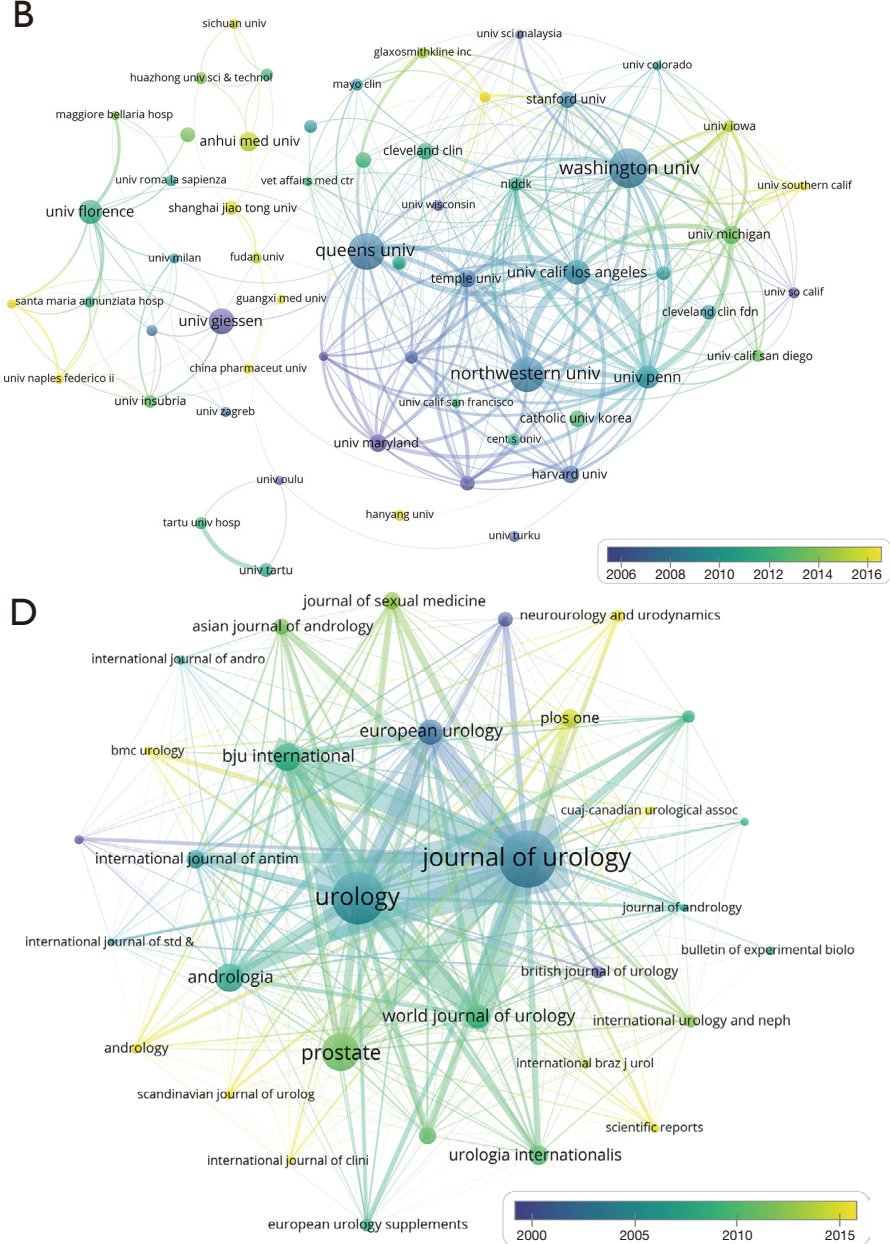

$\mathrm{F}$

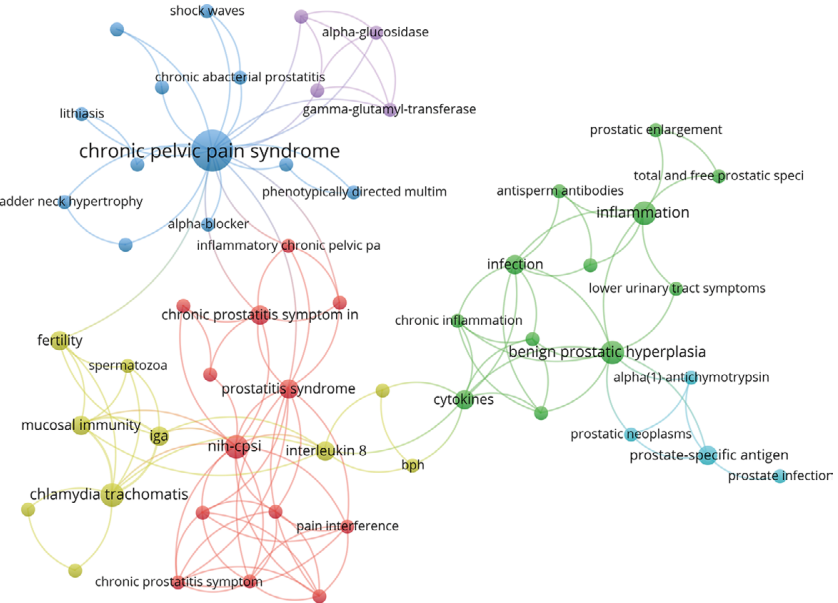

Figure 2 The distribution of countries, institutions, authors and journals on CP/CPPS research. (A) Map of countries with publications on CP/ CPPS. (B) Map of institutions with publications on CP/CPPS. (C) Map of authors with publications on CP/CPPS. (D) Map of journals with publications on CP/CPPS. (E) The keywords of articles about CP/CPPS published by fournal of Urology. (F) The keywords of articles about CP/ CPPS published by European Urology. The nodes represent the countries, institutions, authors and journals. The depth of color represents the publication year of the included articles. The size of the dot represents the number of publications. The thickness of the connecting lines represents the strength of collaboration in the countries, institutions, authors and journals. CP/CPPS, chronic prostatitis/chronic pelvic pain syndrome. 
Table 2 The top 10 author contributed to publications about CP/CPPS

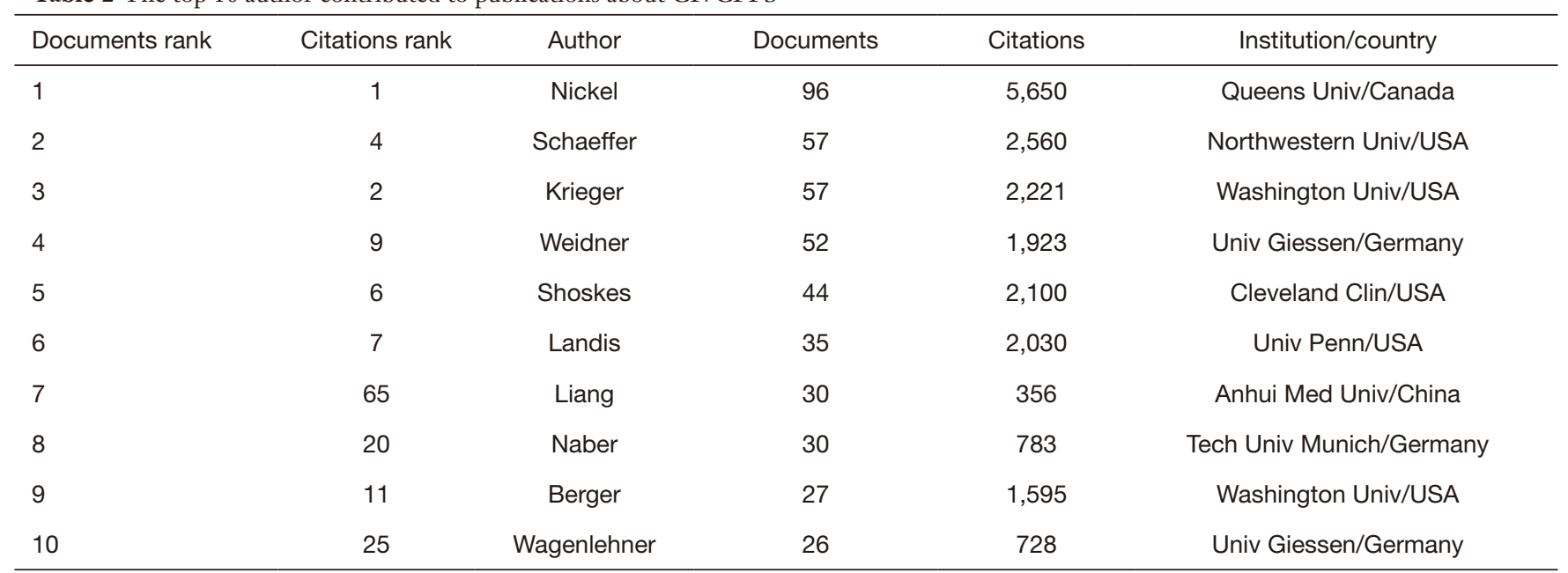

CP/CPPS, chronic prostatitis/chronic pelvic pain syndrome.

Table 3 The top 10 journals and co-cited journals about CP/CPPS

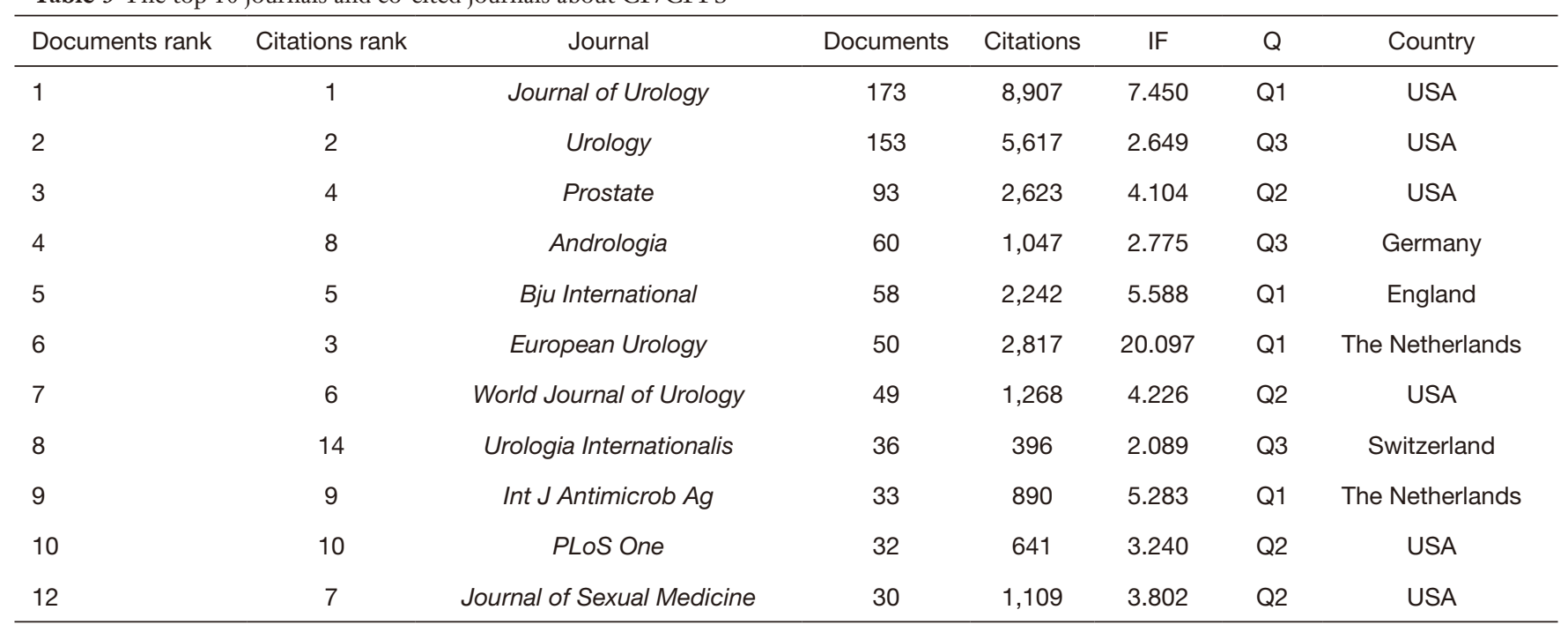

CP/CPPS, chronic prostatitis/chronic pelvic pain syndrome; IF, impact factor; Q, Quartile in Category.

Reference citations, reference cocitation analysis (RCA) and representative articles

The number of reference citations can reflect the core articles in the research field to some extent, and the 1,908 articles were analyzed by the number of reference citations, as shown in Figure 3 A. Most articles $(872 / 1,908$, $45.7 \%$ ) were cited $0 \sim 10$ times, and articles $(56 / 1,908,2.9 \%)$ cited greater than or equal to $100(\mathrm{~T} \geq 100)$ were used to construct the cooccurrence network map, as shown in Figure 3B. The top 20 papers (9-28) (cited greater than or equal to 141) according to the number of citations were analyzed and are shown in Table 4.

RCA expresses the relationship between articles by frequently cited articles; the more frequently the two articles have been cocited, the stronger their linkage is (29). Therefore, RCA is usually used to explore research hotspots in a given academic field, which explores the cocitation relevance between articles and generalizes these data to create major clusters. A knowledge map of cocitation articles is shown in Figure $3 C$. Article cocitation was 

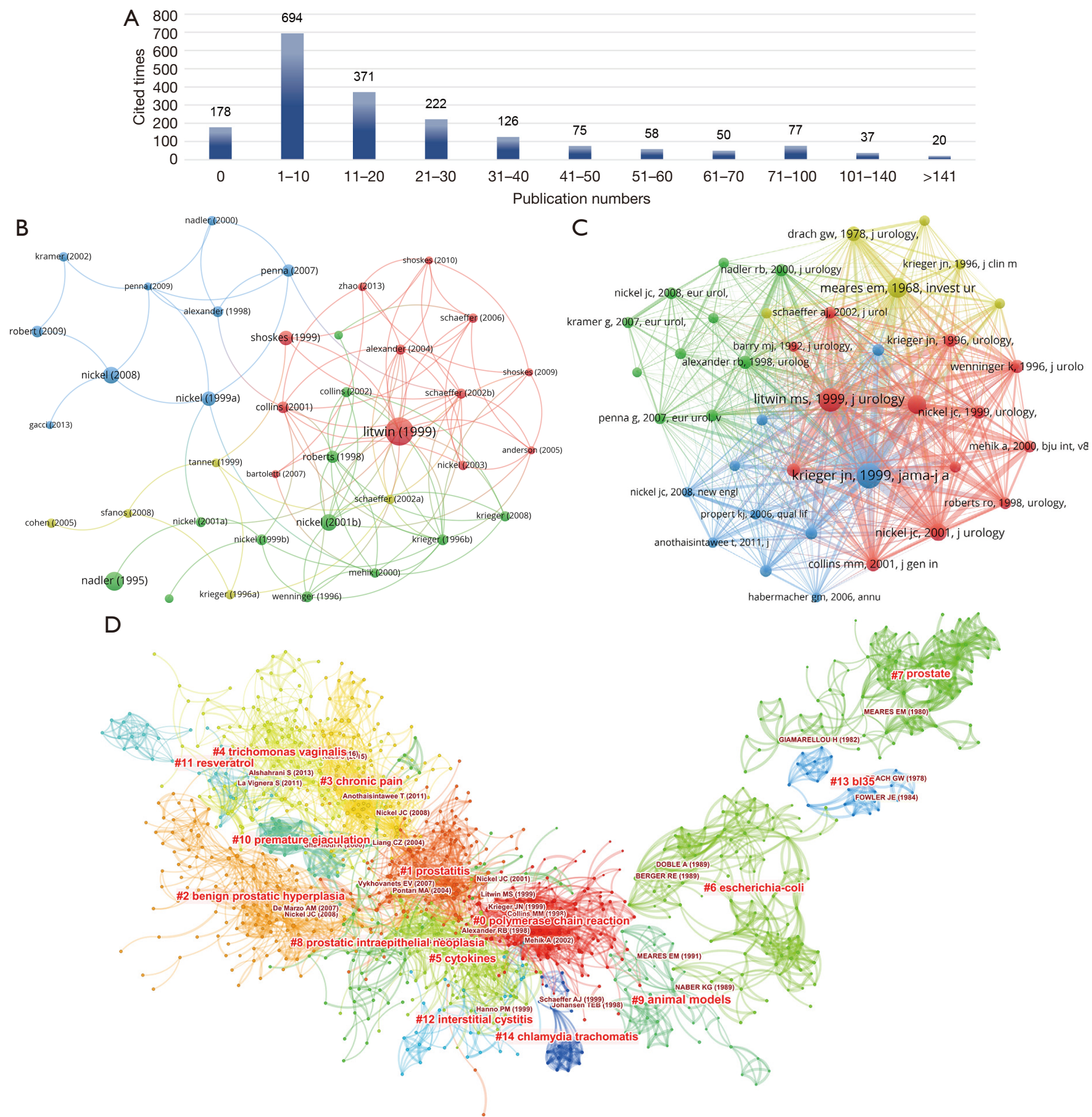

Figure 3 Reference citations and RCA of CP/CPPS research. (A) Analysis of citation times of references on CP/CPPS. (B) Map of the analysis of cocited references on CP/CPPS. (C) Map of RCA on CP/CPPS. (D) The cluster map of RCA on CP/CPPS. RCA, reference cocitation analysis; $\mathrm{CP} / \mathrm{CPPS}$, chronic prostatitis/chronic pelvic pain syndrome. 
Table 4 The top 20 highly cited articles about CP/CPPS

\begin{tabular}{|c|c|c|}
\hline Rank & Articles & Citations \\
\hline 1 & Litwin [1999] (9) & 576 \\
\hline 2 & Nadler [1995] (10) & 318 \\
\hline 3 & Nickel [2001c] (11) & 273 \\
\hline 4 & Nickel [2008b] (12) & 272 \\
\hline 5 & Nickel [1999a] (13) & 223 \\
\hline 6 & Shoskes [1999a] (14) & 221 \\
\hline 7 & Penna [2007b] (15) & 177 \\
\hline 8 & Roberts [1998] (16) & 176 \\
\hline 9 & McNaughton Collins [2001b] (17) & 164 \\
\hline 10 & Robert [2009b] (18) & 162 \\
\hline 11 & Screponi [2001] (19) & 157 \\
\hline 12 & Wenninger [1996] (20) & 157 \\
\hline 13 & Krieger [1996b] (21) & 153 \\
\hline 14 & Alexander [1998] (22) & 150 \\
\hline 15 & Nickel [1999b] (23) & 149 \\
\hline 16 & Collins [2002] (24) & 146 \\
\hline 17 & Krieger [2008] (25) & 144 \\
\hline 18 & Schaeffer [2002c] (26) & 144 \\
\hline 19 & Nickel [2001a] (27) & 142 \\
\hline 20 & Alexander [2004] (28) & 141 \\
\hline
\end{tabular}

$a, b, c$, in the table are the results of software, to distinguish different papers published by the author in the same year. CP/ CPPS, chronic prostatitis/chronic pelvic pain syndrome.

clustered into 11 hotspots, as shown in Figure 3D.

\section{Analysis of keywords}

There were 2,675 keywords in 1,908 articles that were analyzed. For a better understanding of the relationship among them, the keywords meaning to correlative subject headings were removed from the list, and the keywords with similar meanings were merged for analysis. The frequencies of keywords greater than or equal to $8(\mathrm{~T} \geq 8)$ were used to construct the co-occurrence network map, as shown in Figure $4 A$. A cluster analysis for keywords was performed using the K-means algorithm, and it resulted in 12 clusters, as shown in Figure $4 B$. The value of $\mathrm{S}$ is 0.7421 , and an $\mathrm{S}$ value greater than 0.5 indicates reasonable clustering.
Stratified analyses were performed to analyze the strongest keyword burst according to the year of publication. The strongest keyword bursts were analyzed according to the year of publication, as shown in Figure $4 C$.

\section{Discussion}

Based on 1970-2020 data in WoS, a total of 1,908 articles were published in 476 journals, with 31,723 cited references by 1,905 institutions from 71 countries/regions. The knowledge domain and emerging trends in CP/CPPS have been analyzed by scientometric research based on VOSviewer and CiteSpace to offer a better understanding of CP/CPPS development in the past 50 years for researchers.

The annual output of CP/CPPS-related publications has been generally increasing in the past 50 years, and almost 120 studies will be published in 2021 according to linear fitting, as shown in Figure $2 A$. The USA, Canada, China, Italy and Germany were the top 5 productive countries, and the USA was the foremost productive country. Cooperations existed among the countries, but the cooperation between the USA and Canada was the closest, followed by the USA and China and the USA and Italy. According to the timeline of Figure $2 \mathrm{~A}$, the countries that are still active in recent years on CP/CPPS research are China, followed by South Korea, France and Belgium.

A total of 1,908 publications about CP/CPPS were published by 1,905 institutions, 7,538 authors, and $1,449(1,449 / 1,905,76.1 \%)$ institutions. A total of 6,207 $(6,207 / 7,538,82.3 \%)$ authors published only 1 paper, showing that CP/CPPS research performs broadly but scattered and not in-depth. However, CP/CPPS research still forms a stable research team at present, countries with a large number of publications have core institutions, and the core institutions have their own representative authors. Although Queen's University (Canada) has a slightly smaller number of articles than Washington University (USA), it has the most citations and closer associations with other institutions and should be considered the core institution on CP/CPPS research. There is no doubt that Prof. Nickel at Queen's University is the most active author, with the most publications, citations and collaborations. Fournal of Urology, the official journal of the American Urological Association (AUA), was the most productive journal, and as the official journal of the European Association of Urology (EAU), European Urology was the journal with the highest IF in the field of CP/CPPS. We analyzed the two journals and found that Fournal of Urology focus on pain and inflammation, 


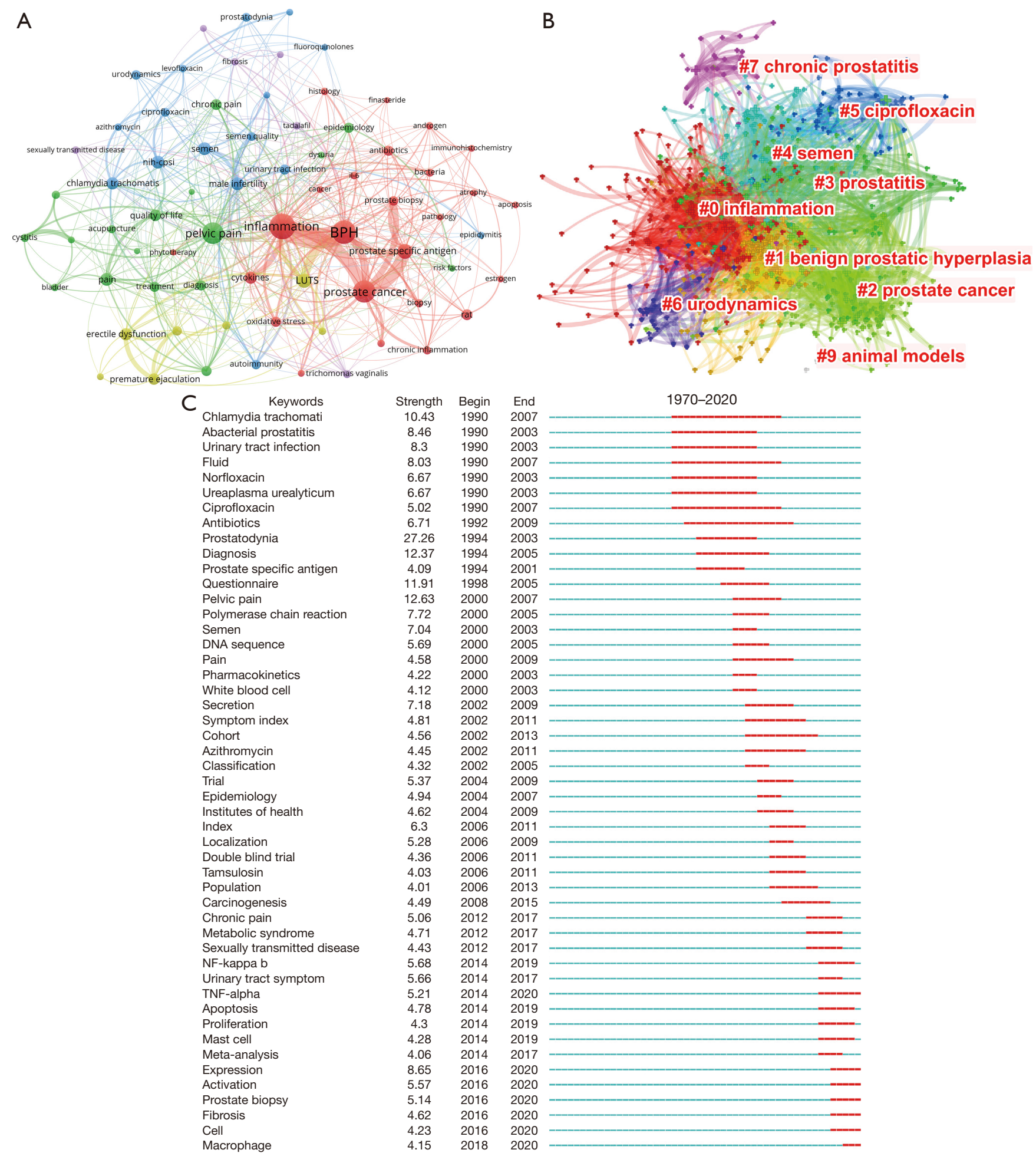

Figure 4 Analysis of keywords on CP/CPPS. (A) The co-occurrence analysis of keywords on CP/CPPS. (B) The cluster map of keywords on CP/CPPS. (C) The burst keywords of keywords on CP/CPPS. The nodes represent the keywords. The depth of color represents the topic of keywords. The size of the dot represents the number of keywords. The thickness of the connecting lines represents the strength of the correlation in the keywords. CP/CPPS, chronic prostatitis/chronic pelvic pain syndrome. 
European Urology seems have more interested in NIHCPSI, and the correlation between CP/CPPS with benign prostatic hyperplasia $(\mathrm{BPH})$ and prostate specific antigen (PSA).

According to the reference citations, approximately half of the literature was cited $0-10$ times, and 20 articles were cited more than 140 times. These studies have a high degree of authority and credibility, so they should be given adequate attention. The theme mainly concerns expert opinions or consensus, epidemiology, drug therapy, comorbidities or related complications. The literature on NIH-CPSI published by Litwin et al. (9) in 1999 is the core paper in the field of CP/CPPS, reflecting that NIHCPSI has been widely accepted as a criterion for CP/CPPS efficacy.

The publications with strong citation bursts were followed closely by the researchers over a time period. According to RCA and keywords, the emerging topics about epidemiology and clinical and experimental studies over the past 50 years were summarized. We find that the focus of the present research on CP/CPPS mainly involved inflammation, pelvic pain, lower urinary tract symptoms (LUTS), quality of life, and other clinical symptoms. It can be found that the main direction of CP/CPPS is still focus around clinical symptoms, interestingly, this direction is also being followed by the treatment of CP/CPPS. Clinicians tend to use symptomatic treatment, and CP/CPPS is mainly treated by antibiotics, alpha receptor blockers, nonsteroidal antiinflammatory drugs and other treatment methods at present (12). Treatment or drug selection was often chosen according to patients willingness and doctors experience, but the efficacy is unstable and symptoms are prone to relapse, and the non-standard use of antibiotics easily cause some side effects and the evolution of antibiotic resistance (11). Therefore, new therapeutic strategies and/or new adjuvant drugs still need to be explored. Similarly, we could find that epidemiological research on CP/CPPS also mainly focused on cross-sectional and cohort studies about the clinical symptoms and drugs. We found that researchers had also focused on other diseases or clinical indicators that CP/CPPS may cause or often combine with, such as the correlation with $\mathrm{BPH}$, prostate cancer, premature ejaculation, erectile dysfunction, PSA and semen quality, as shown in the Figure 4. This seems to be an interesting research direction for further work and worth exploring, based on the background of high incidence of CP/CPPS in men at present. Experimental studies mainly involved animal models, research on the pathological mechanism and drugs. This corresponds with the present findings.

Throughout the history of CP/CPPS in the past 50 years, we found that research on inflammation has always been the focus of research, but we could still find that there are different emphases in different stages. The perceptions of CP/CPPS have gradually the trend of changing from inflammation and infection to chronic pain (30). The fourcup method was proposed by Meares and Stamey in 1968 as the classification method of prostatitis, and the two-cup method was proposed by Nickel in 1998 as the optimization method of bacterial and nonbacterial prostatitis (31). Then, according to the classification method proposed by the NIH in 1995, the positioning of bacterial culture, pain and sterility were placed more emphasis on as the clinical features of CP/CPPS. In addition, since 1999, Litwin et al. (9) launched the NIH-CPSI scale. The CP/CPPS evaluation standard has gradually changed from routine laboratory tests such as expressed prostatic secretions to NIH-CPSI, meaning that treatment strategy is based on improving symptoms.

At present, although a great deal of work has investigated the mechanism of CP/CPPS, clinical treatment mainly focuses on symptomatic improvement, including alleviating pain and relieving urination symptoms, thus improving the quality of life of patients (32). Therefore, CP/CPPS treatment mainly aims at alleviating pain, improving urination symptoms and improving the quality of life of patients and focuses on symptomatic treatment to improve symptoms (33). Although a large number of mechanisms and drug studies have been carried out, most drugs are still empirically used. Therefore, there is an urgent requirement to discover novel therapeutic targets and develop drugs or novel therapeutics for the treatment of CP/CPPS.

\section{Conclusions}

We used VOSviewer and CiteSpace to analyze the knowledge base and research hotspots on CP/CPPS publications from 1970 to 2020 . The USA contributed the most to the CP/CPPS research, and the University of Washington (USA) and Queen's University (Canada) are the core institutions. Prof. Nickel is the core researcher. fournal of Urology and Urology are the significant journals for CP/CPPS. Epidemiology and clinical research on inflammation and chronic pain are recent major topics in CP/CPPS research. Exploring the microscopic mechanism and developing new therapeutic strategies to prevent $\mathrm{CP} /$ CPPS are recent topics in research. This scientometric 
review offers a comprehensive understanding of CP/CPPSrelated publications from 1970 to 2019, which could supply references to researchers in this field. As investigators discover more information on CP/CPPS, we expect that the prevention, diagnosis, management, and prognosis of $\mathrm{CP} /$ CPPS will soon become more effective and efficient.

\section{Acknowledgments}

Funding: This work was supported by the National Natural Science Foundation of China (No. 82104880) and Scientific and Technological Innovation Project of China Academy of Chinese Medical Sciences (No. CI2021A02208).

\section{Footnote}

Conflicts of Interest: All authors have completed the ICMJE uniform disclosure form (available at https://apm. amegroups.com/article/view/10.21037/apm-21-3068/coif). The authors have no conflicts of interest to declare.

Ethical Statement: The authors are accountable for all aspects of the work in ensuring that questions related to the accuracy or integrity of any part of the work are appropriately investigated and resolved.

Open Access Statement: This is an Open Access article distributed in accordance with the Creative Commons Attribution-NonCommercial-NoDerivs 4.0 International License (CC BY-NC-ND 4.0), which permits the noncommercial replication and distribution of the article with the strict proviso that no changes or edits are made and the original work is properly cited (including links to both the formal publication through the relevant DOI and the license). See: https://creativecommons.org/licenses/by-nc-nd/4.0/.

\section{References}

1. Franco JV, Turk T, Jung JH, et al. Pharmacological interventions for treating chronic prostatitis/chronic pelvic pain syndrome. Cochrane Database Syst Rev 2019;10:CD012552.

2. Franco JVA, Turk T, Jung JH, et al. Pharmacological interventions for treating chronic prostatitis/chronic pelvic pain syndrome: a Cochrane systematic review. BJU Int 2020;125:490-6.

3. Magistro G, Wagenlehner FM, Grabe M, et al. Contemporary Management of Chronic Prostatitis/
Chronic Pelvic Pain Syndrome. Eur Urol 2016;69:286-97.

4. Doiron RC, Shoskes DA, Nickel JC. Male CP/CPPS: where do we stand? World J Urol 2019;37:1015-22.

5. Chen C, Song M. Visualizing a field of research: A methodology of systematic scientometric reviews. PLoS One 2019;14:e223994.

6. Ioannidis JP, Boyack KW, Small H, et al. Bibliometrics: Is your most cited work your best? Nature 2014;514:561-2.

7. van Eck NJ, Waltman L. Software survey: VOSviewer, a computer program for bibliometric mapping. Scientometrics 2010;84:523-38.

8. Chen C. CiteSpace II: Detecting and visualizing emerging trends and transient patterns in scientific literature. J Am Soc Inf Sci Technol 2006;57:359-77.

9. Litwin MS, McNaughton-Collins M, Fowler FJ Jr, et al. The National Institutes of Health chronic prostatitis symptom index: development and validation of a new outcome measure. Chronic Prostatitis Collaborative Research Network. J Urol 1999;162:369-75.

10. Nadler RB, Humphrey PA, Smith DS, et al. Effect of inflammation and benign prostatic hyperplasia on elevated serum prostate specific antigen levels. J Urol 1995;154:407-13.

11. Nickel JC, Downey J, Hunter D, et al. Prevalence of prostatitis-like symptoms in a population based study using the National Institutes of Health chronic prostatitis symptom index. J Urol 2001;165:842-5.

12. Nickel JC, Roehrborn CG, O'Leary MP, et al. The relationship between prostate inflammation and lower urinary tract symptoms: examination of baseline data from the REDUCE trial. Eur Urol 2008;54:1379-84.

13. Nickel JC, Downey J, Young I, et al. Asymptomatic inflammation and/or infection in benign prostatic hyperplasia. BJU Int 1999;84:976-81.

14. Shoskes DA, Zeitlin SI, Shahed A, et al. Quercetin in men with category III chronic prostatitis: a preliminary prospective, double-blind, placebo-controlled trial. Urology 1999;54:960-3.

15. Penna G, Mondaini N, Amuchastegui S, et al. Seminal plasma cytokines and chemokines in prostate inflammation: interleukin 8 as a predictive biomarker in chronic prostatitis/chronic pelvic pain syndrome and benign prostatic hyperplasia. Eur Urol 2007;51:524-33; discussion 533.

16. Roberts RO, Lieber MM, Rhodes T, et al. Prevalence of a physician-assigned diagnosis of prostatitis: the Olmsted County Study of Urinary Symptoms and Health Status Among Men. Urology 1998;51:578-84. 
17. McNaughton Collins M, Pontari MA, O'Leary MP, et al. Quality of life is impaired in men with chronic prostatitis: the Chronic Prostatitis Collaborative Research Network. J Gen Intern Med 2001;16:656-62.

18. Robert G, Descazeaud A, Nicolaïew N, et al. Inflammation in benign prostatic hyperplasia: a 282 patients' immunohistochemical analysis. Prostate 2009;69:1774-80.

19. Screponi E, Carosa E, Di Stasi SM, et al. Prevalence of chronic prostatitis in men with premature ejaculation. Urology 2001;58:198-202.

20. Wenninger K, Heiman JR, Rothman I, et al. Sickness impact of chronic nonbacterial prostatitis and its correlates. J Urol 1996;155:965-8.

21. Krieger JN, Egan KJ, Ross SO, et al. Chronic pelvic pains represent the most prominent urogenital symptoms of "chronic prostatitis". Urology 1996;48:715-21; discussion 721-2.

22. Alexander RB, Ponniah S, Hasday J, et al. Elevated levels of proinflammatory cytokines in the semen of patients with chronic prostatitis/chronic pelvic pain syndrome. Urology 1998;52:744-9.

23. Nickel JC, Nyberg LM, Hennenfent M. Research guidelines for chronic prostatitis: consensus report from the first National Institutes of Health International Prostatitis Collaborative Network. Urology 1999;54:229-33.

24. Collins MM, Meigs JB, Barry MJ, et al. Prevalence and correlates of prostatitis in the health professionals followup study cohort. J Urol 2002;167:1363-6.

25. Krieger JN, Lee SW, Jeon J, et al. Epidemiology of prostatitis. Int J Antimicrob Agents 2008;31 Suppl 1:S85-90.
26. Schaeffer AJ, Landis JR, Knauss JS, et al. Demographic and clinical characteristics of men with chronic prostatitis: the national institutes of health chronic prostatitis cohort study. J Urol 2002;168:593-8.

27. Nickel JC, True LD, Krieger JN, et al. Consensus development of a histopathological classification system for chronic prostatic inflammation. BJU Int 2001;87:797-805.

28. Alexander RB, Propert KJ, Schaeffer AJ, et al. Ciprofloxacin or tamsulosin in men with chronic prostatitis/chronic pelvic pain syndrome: a randomized, double-blind trial. Ann Intern Med 2004;141:581-9.

29. Büttner F, Ardern CL, Blazey P, et al. Counting publications and citations is not just irrelevant: it is an incentive that subverts the impact of clinical research. Br J Sports Med 2021;55:647-8.

30. Anothaisintawee T, Attia J, Nickel JC, et al. Management of chronic prostatitis/chronic pelvic pain syndrome: a systematic review and network meta-analysis. JAMA 2011;305:78-86.

31. Nickel JC, Shoskes D, Wang Y, et al. How does the premassage and post-massage 2-glass test compare to the Meares-Stamey 4-glass test in men with chronic prostatitis/ chronic pelvic pain syndrome? J Urol 2006;176:119-24.

32. Magri V, Marras E, Restelli A, et al. Multimodal therapy for category III chronic prostatitis/chronic pelvic pain syndrome in UPOINTS phenotyped patients. Exp Ther Med 2015;9:658-66.

33. Kessler TM. Chronic Pelvic Pain Syndrome: Light at the End of the Tunnel? Eur Urol 2016;69:298-9.
Cite this article as: Liu SJ, Gao QH, Deng YJ, Zen Y, Zhao M, Guo J. Knowledge domain and emerging trends in chronic prostatitis/chronic pelvic pain syndrome from 1970 to 2020: a scientometric analysis based on VOSviewer and CiteSpace. Ann Palliat Med 2022;11(5):1714-1724. doi: 10.21037/ apm-21-3068 